\title{
Characterization, morphology, and biodegradation of bioplastic fertilizer (BpF) composites made of poly(butylene succinate) blended with oil palm biomass and fertilizer
}

\begin{abstract}
Poly(butylene succinate) (PBS) is a versatile biodegradable polymer that can be processed into slow-release bioplastic fertilizer $(\mathrm{BpF})$ composites using twin screw extruder extrusion method, with controlled formulation and temperature. In this study, slow-release BpF composites were created by blending NPK fertilizer with biodegradable plastic composites and oil palm biomass. Temperature processing was done at $125^{\circ} \mathrm{Cï} 145^{\circ} \mathrm{C}$ for $3 \ddot{i} 5 \mathrm{~min}$ using twin screw extruder. Its thermal degradation occurred initially at $263.44^{\circ} \mathrm{C}$ and reached maximum at $300.73^{\circ} \mathrm{C}$. In biodegradation test, the weight losses of PBS/NPKC1 and PBS/NPKC2 were about $60 \%$ while the weight losses of PBS/EFB/NPKC1 and $\mathrm{PBS} / \mathrm{EFB} / \mathrm{NPKC} 2$ were $72.68 \%$ and $73.09 \%$, respectively. It was observed under scanning electron microscope that PB1 and PB2 showed more homogeneous adhesion and better wetting of PBS.
\end{abstract}

Keyword: Poly(butylene succinate); Bioplastic fertilizer (BpF); Oil palm biomass; Fertilizer 